Appl. Ent. Zool. 16 (2) : $79-87$ (1981)

\title{
Mode of Action of Bacillus thuringiensis $\delta$-Endotoxin : Effect on Galleria mellonella (Lepidoptera : Pyralidae)
}

\author{
Junko Nishitssutsuji-Uwo and Yasuhisa Endo \\ Shionogi Research Laboratories, Shionogi \& Co., Ltd., \\ Fukushima-ku, Osaka 553, Japan
}

(Received October 24, 1980)

\begin{abstract}
The general pathology of Galleria mellonella induced by Bacillus thuringiensis $\delta$-endotoxin was studied in terms of symptoms, relative roles of crystals and spores, hemolymph chemistry and the associated histopathology of the midgut. Bioassay using pure crystals and spores clearly demonstrated that the primary cause of death of Galleria larvae was the crystals and the presence of spores was only supplemental. Four hours after a single administration of crystals, changes appearing in the columnar cells of midgut epithelium were very similar to those observed in completely paralyzed Bombyx mori; cells swelled losing their microvilli, burst or sloughed off into the lumen. Changes appearing in the goblet cells were different from those observed in Bombyx mori; mitochondria came out from cytoplasmic projections which diminished in number in some cells. When cells sloughed off from the basement lamina, the projections were totally deformed. Mitosis and development of new columnar and goblet cells to replace the broken cells were more frequently observed in the regenerative cell-masses (nidi) than in those of normal epithelium. Although blood $\mathrm{pH}$ scarcely changed, the $\mathrm{K}^{+}$level rose up to 1.3 times. These results led to the conclusion that the reaction of $G$. mellonella to serotype VII is not that of a Type III host species.
\end{abstract}

\section{INTRODUGTION}

In previous papers we have extensively studied the mode of $\delta$-endotoxin action on the so-called Type I (Bombyx mori) and Type II (Pieris rapae, Lymantria dispar and Ephestia cautella) insects. We have found the general pathology in terms of larval behavior, the relative role of crystals and spores, hemolymph chemistry and the associated histopathology (Nishirtsutsujr-Uwo and Endo, 1890a, b; ENDo and Nishirtsutsuji-Uwo, 1980; and unpublished data) and found that there are sequential differences from species to species in any account of the disease induced by Bacillus thuringiensis intoxication.

We are now concerned with studies on so-called Type III insect which is said to die by the ingestion of the toxin, only in the presence of spores (Heimpel and ANGus, 1959). So far, two species of Lepidoptera, Ephestia (Anagasta) kuehniella and Galleria mellonella, have been described as Type III insects. Curiously Ephestia kueniella is closely related to $E$. cautella and yet the former has been classified as a Type III and the latter as a Type II insect (MaGaughey, 1978). However, the absolute requirement of both crystals and spores to cause mortality has been demonstrated in Galleria mellonella by Burges et al. (1976).

Thus, we planed a thorough study of $\delta$-endotoxin intoxication on Galleria mel- 
lonella as representative Type III insect. This paper describes the results of bioassay, hemolymph chemistry and the associated histopathology of the midgut. Our results clearly demonstrated that $G$. mellonella is not a Type III insect in its reaction to serotype VII because the crystals alone were active enough to kill the larvae of this species.

\section{MATERIALS AND METHODS}

Bacteria. Bacillus thuringiensis (B.t) subsp. aizawai (serotype VII; esterase type, galleriae) and its sporeless mutant strain I-45 (Nishirtsutsuji-Uwo et al., 1975) were used in the present experiments. The original strain was used as a source of spores and the mutant strain as a source of crystals. The bacterial cultivation method was described elsewhere (Nishirtsutsuji-Uwo et al., 1979). Autolyzed cultures were washed by the method of Delafield et al. (1968) and residual cells and cell debris were removed by the method of Sacks and Alderton (1961) (phase mixture 1) and extraction was repeated many times. The upper and middle phases contained spores and crystals in the original strain, and crystals and a small amount of debris in the mutant strain.

Spores were separated from crystals by the method of Goodman et al. (1967) with 15-20 repetitions of the procedure. The upper phase contained spores with $0.1 \%$ contamination with crystals. Grystal-free spores were obtained by extracting the contaminating crystals with partially purified enzymes prepared from silkworm gut juice as described in a previous paper (Nishitsutsuji-Uwo and Endo, 1980b). Absolutely crystal-free spores were employed in the present experiment.

A crystal preparation obtained from a mutant strain sometimes contained a few small unknown spherical bodies which may have been derived from degenerated forespores (Nishimura and Nishirsutsuji-Uwo, 1980). Although plate counts showed no viable spore in this preparation, these contaminants were removed by the method of Goodman et al. (1967).

Insects and bioassay. Stock cultures (gifts from Dr. J. Mitsuhashi of National Inst. of Agricultural Science) of the wax moth, Galleria mellonella, were reared at $30^{\circ} \mathrm{C}$ either on an artificial diet or on honey comb, followed by adaptation to an artificial diet, in most cases, a few days before the test. No difference due to food was observed in the obtained results.

The artificial diet, prepared by the method of Burges et al. (1976), comprised of $33 \%$ baby cereal (Gerber, high protein cereal No. 727), 23\% clear honey, $21 \%$ glycerine, $15 \%$ debittered yeast powder and $8 \%$ water. Five grams of the artificial diet was uniformally spreaded in the bottom of a square plastic case $(55 \times 50 \times 12 \mathrm{~mm})$. Next, $0.5 \mathrm{ml}$ of suspension containing either pure crystals, pure spores or crystal-spore mixtures $(1: 1)$ was applied homogeneously on the surface of the food $(\sim 2 \mathrm{~mm}$ in thickness) and dried. For the control series, $0.5 \mathrm{ml}$ of $\mathrm{H}_{2} \mathrm{O}$ without toxin was applied. Ten larvae 15 days old (50-60 mg body wt., mainly fourth instars) were then transferred into each case and kept at $30^{\circ} \mathrm{C}$. Daily observation was continued for 7 days. All experiments repeated 7-8 times with nine serial dilutions. Galleria larvae occasionally fed on each other $(2-3 \%)$. Since the color of the body killed by $\delta$-endotoxin changes to black, we judged the death by toxin only when the left-over body was black. 
Determination of $p H$ and ion concentration. A pure crystal suspension (20 $\mu 1$ containing either 200,100 or $50 \mu \mathrm{g}$ crystals) was injected per os into a sixth-instar larva (150 mg body wt.). Blood was collected and pooled from 7-8 living larvae each (double sampling, total 15 larvae) with a glass capillary inserted into a wound made an abdominal leg at 0,4 and $24 \mathrm{hr}$ after the injection. Midgut juice was obtained after the dissection of the midgut of 100 normal larvae and pooled.

The $\mathrm{pH}$ was measured using micro-combination glass $\mathrm{pH}$ probes, MI-410 (Microelectrodes Inc.), and ion concentrations were determined (courtesy of $\mathrm{Mr}$. $\mathrm{T}$. DozAkI of our Laboratories) using an atomic absorption spectrophotometer NF-1B (Toshiba-Beckman).

Histopathology of the midgut. At 4 and $24 \mathrm{hr}$ after the injection of the crystal suspension $(200,100$ and $50 \mu \mathrm{g})$ per os into a sixth-instar larva, seven midguts each were fixed. For light microscopy (four midguts), the whole midgut was fixed with BouIn's fixative for $2 \mathrm{hr}$. Serial paraffin sections were stained with MAYER's acid hemalaum and eosin.

For electron microscopy (three midguts), the anterior-middle region of midgut was excised and fixed with $2.5 \%$ buffered glutaraldehyde for $1 \mathrm{hr}$ at $4{ }^{\circ} \mathrm{C}$, followed by the postfixation with $1 \% \mathrm{OsO}_{4}$ for $1 \mathrm{hr}$ at $4^{\circ} \mathrm{C}$. After the dehydration in ethanol series, the tissues were embedded in Epon 812. Sections were made with an LKB8800 microtome, contrasted with uranyl acetate and lead hydroxide, and examined with a JEM-100 CX electron microscope.

\section{RESULTS AND DISGUSSION}

Symptoms and insecticidal effects of crystal and spore

Even after administration of a lethal dose of toxin, larvae act normally for 3-5 days until death. Cessation of feeding may not occur. Occasional diarrhea was sometimes observed.

Pure preparations of crystals, spores and their $1: 1$ mixtures of B.t serotype VII were tested against 15 day-old larvae of Galleria mellonella. Within $48 \mathrm{hr}$ larvae

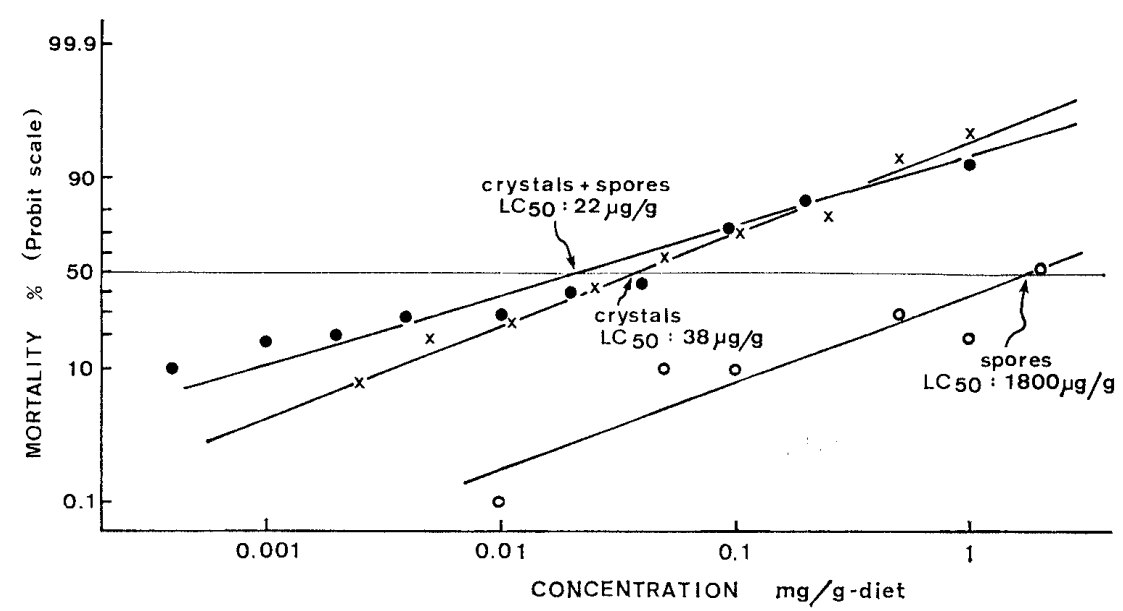

Fig. 1. Toxicity of crystals and/or spores of Bacillus thuringiensis (serotype VII) to Galleria mellonella over 7 days. Each point shows the average value of mortality obtained from $7-8$ repeated assays. 
showed little mortality which was also characteristic of Pieris rapae and Lymantria dispar as reported previously (Nishirtsutsuji-Uwo and Endo, 1980b). Most of the larvae died in 3-4 days after the impact of the toxin. The peak day of mortality came earlier than that of Ephestia cautella which was over 5 days. In general, the susceptibility of Galleria larvae to killing by B.t was extremely low - it was about a hundred times less than that of Bombyx larvae, yet five times higher than that of Ephestia cautella.

Pure crystals were found to be toxic to larvae of this insect. Spores alone also killed it, although their activity was 1/50 that of the crystals. Crystal-spore mixtures showed a four fold (based on crystal weight) or two fold (based on mixture wt.) higher effect than the crystals alone. The results are shown in Fig. 1. Similar results have been obtained with Pieris rapae, Lymantria dispar, Ephestia cautella (Nishirtsutsuji-Uwo and ENDO, 1980b) and Choristoneura fumiferana (FAST, 1977); all of them are said to be the Type II insects. Our results clearly demonstrated that the primary cause of death of Galleria larvae was the crystals and the presence of spores enhanced crystal toxicity only two to four-fold.

Different results have been obtained by Burges et al. (1976). Their results revealed that crystal-spore mixtures $(1: 1)$ of $B . t$ serotype $\mathrm{V}$ were 10 times more potent than live spores alone and about $10^{4}$ times more potent than crystals alone. They concluded that one or more factors in the spore are at least partly responsible for the potency of serotype $\mathrm{V}$ in $G$. mellonella, which is nearest to that of a Type III host species. We cannot explain these differences in the results. It might be due to differences in the bacterial subspecies employed and in the crystals obtained. Burges et al, obtained crystals, free of live spores by exposing an aliquot of Thuricide to 0.91 Mrad-hr of gamma radiation. As far as our experiments are concerned, G. mellonella does not give a clear Type III response to serotype VII of $B$. thuringiensis.

\section{Histopathology of the midgut epithelium}

The structure of normal midgut epithelium of Galleria larva (Fig. 2) is essentially the same as that seen in other lepidopterous larvae (Endo and Nishirssutsuji-Uwo, 1980; and unpublished data). A distinguishing trait in Galleria is that: Glycogen granules are found as big masses in the cytoplasm of the columnar cells (Fig. 3, GI). The cytoplasmic projections lining the goblet cavity contains a mitochondrion (Fig. 4, M) which is often absent like that seen in Pieris rapae. Epithelial cells of Galleria seem to turn over quickly. Many regenerative cells (Fig. 5) are found in the basal portion of the epithlium as a nidus (a mass of $6-8$ cells) in which mitosis and developing new columnar and goblet cells are often observed. In the portion far from the regenerative cell-mass, we often observe degenerating cells. Basement lamina is sometimes as thick as $20 \mu \mathrm{m}$.

At $4 \mathrm{hr}$ after the forced injection of crystals (either 200, 100 or $50 \mu \mathrm{g} / \mathrm{larva}$ ), columnar cells swelled and protruded by elongation into the gut lumen (Figs. 6, 7). The apical portion was often destroyed with the organella ejected into the lumen. Many cells were torn from the basement lamina. Goblet cells were sometimes pushed out being close to their adjacent elongating columnar cells. Mitosis and development of new cells in nidi became more frequent (Fig. 6, arrows) than those seen in normal epithelium. The injection dose seemed not to make any difference in the degree of histopathological damage.

At the ultrastructural level, disintegration, partial vacuolization and a decrease 


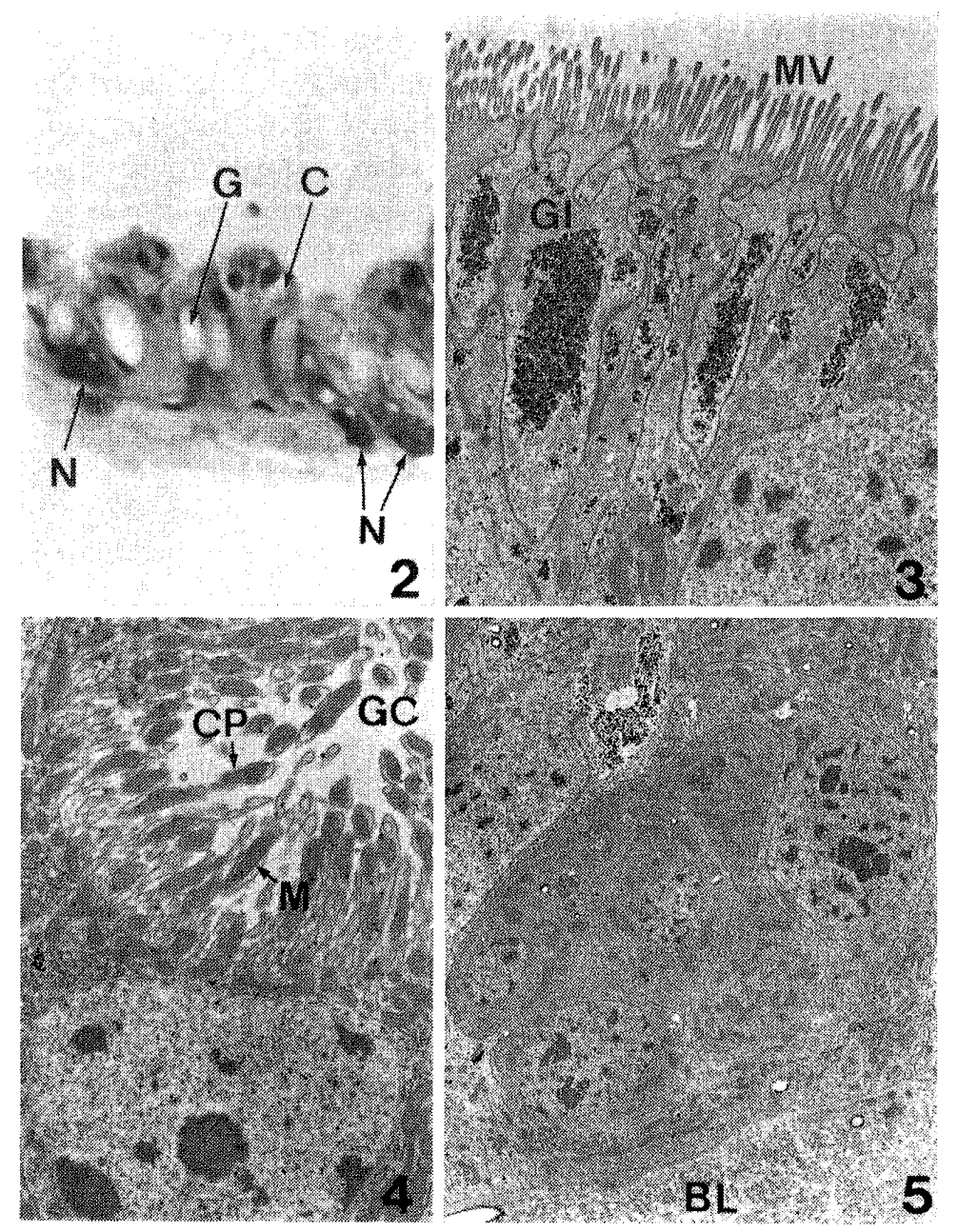

Fig. 2. Light micrograph of normal midgut epithelium of Galleria mellonella (6th-instar larva). C, columnar cell; G, goblet cell; N, nidus of regenerative cells. $\times 370$.

Fig. 3. Electron micrograph of upper portion of normal columnar cells. Numerous microvilli (MV) line at the lumen side. Masses of glycogen granules (Gl) are seen in the cytoplasm. $\times 4,400$.

Fig. 4. Electron micrograph of a part of normal goblet cell. Numerous cytoplasmic projections (GP) line inside the goblet cavity (GC). Most of the projections contain mitochondria $(\mathrm{M}) . \quad \times 6,600$.

Fig. 5. Electron micrograph of normal nidus. Regenerative cells are found in the basal portion of epithelium as a mass or nidus. BL, basement lamina. $\times 2,200$.

in the number or total disappearance of microvilli were observed in the swollen columnar cells (Fig. 7). The compartments formed by basal infoldings of the cell membrane were disintegrated and occasionally disappeared. Glycogen granules obviously diminished in number especially in the center of the mass. Changes appeared in the columnar cells at the 4-hr stage were very similar to those observed in Ephestia cautella at 24-hr exposure and in Bombyx mori at stage 3 paralyzed completely (Endo and Nishitsutsuji-Uwo, 1980; and unpublished data). In the goblet cells, mitochondria came out from projections which were sometimes totally empty (Fig. 8). Projections occasionally diminished in number or disintegrated. The endo- 
plasmic reticulum and basal infoldings were usually preserved intact. In the case where goblet cells were sloughed off from the basement lamina, cytoplasmic projections began to change into vacuole-like structure and became totally deformed in the later stage (Fig. 9). Cells burst and the cytoplasm became scanty. Such changes were also observed in some goblet cells of Lymantria dispar (unpublished data).

At $24 \mathrm{hr}$ after the injection of the crystals, histopathological damages were the same as, or rather lesser than those seen at the 4-hr stage because of new cell regeneration (Fig. 10). Mitosis continued to proceed vigorously in the nidi. In the gut
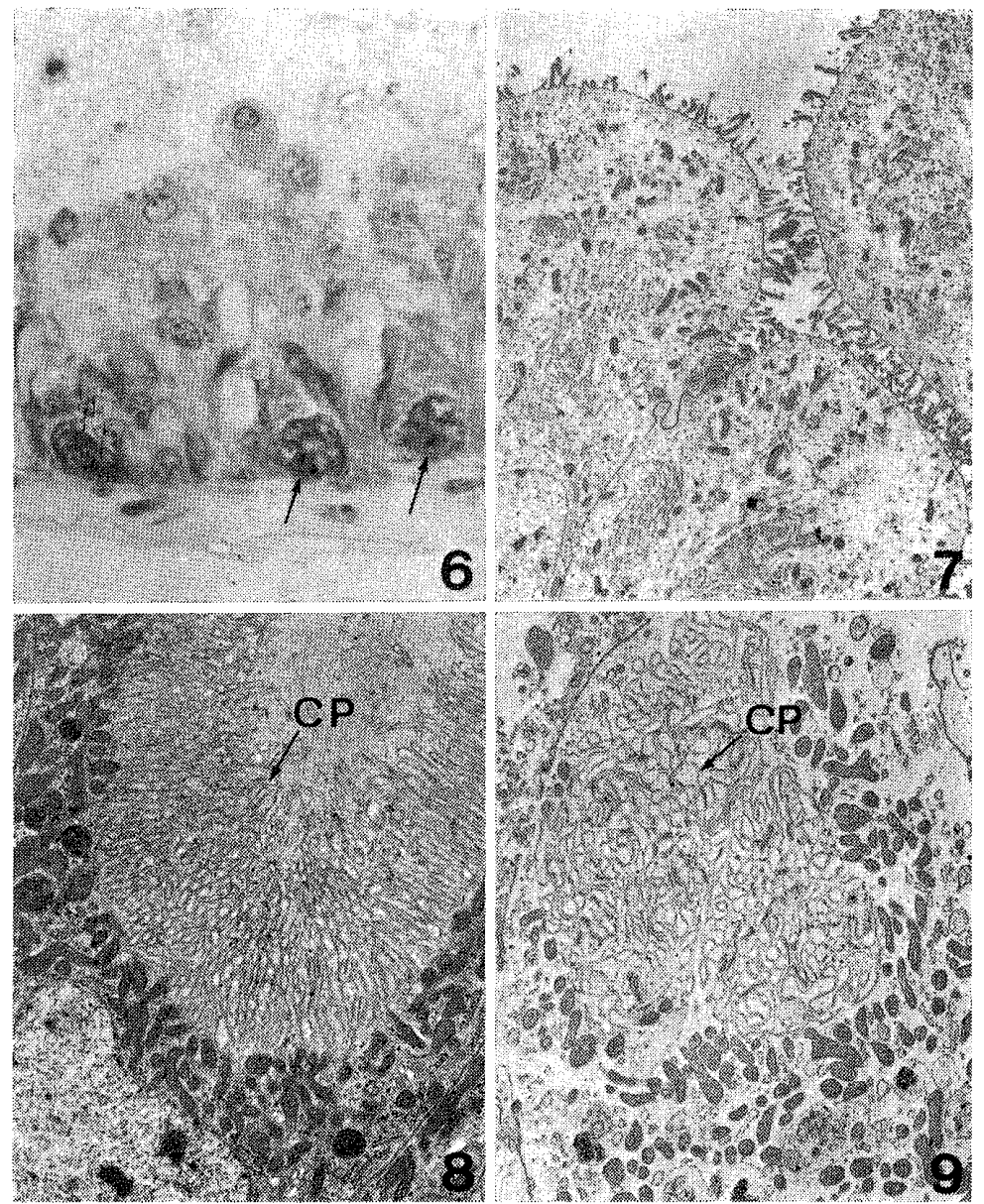

Fig. 6. Light micrograph of midgut epithelium at $4 \mathrm{hr}$ after the forced injection of crystals $(200 \mu \mathrm{g} /$ larva $)$. Epithelial cells swell and elongate into the lumen. Some cells are sloughed into the lumen of midgut. Mitoses of regenerative cells are frequently seen (arrows). $\times 370$.

Fig. 7. Electron micrograph of upper portion of columnar cells at $4 \mathrm{hr}$ after the forced injection of crystals ( $50 \mu \mathrm{g} /$ larva). Apices of columnar cells swell and their microvilli disintegrate. $\times 2,100$.

Fig. 8. Electron micrograph of a part of goblet cell at $4 \mathrm{hr}$ after the forced injection of crystals (50 $\mu \mathrm{g} /$ larva). Mitochondria come out from cytoplasmic projections (CP), which are entirely empty. $\times 5,200$.

Fig. 9. Electron micrograph of a part of disrupted goblet cell at $4 \mathrm{hr}$ after the forced injection of crystals $(200 \mu \mathrm{g} /$ larva). Cytoplasmic projections (CP) disintegrate and change into vacuole-like structure. $\times 4,000$. 


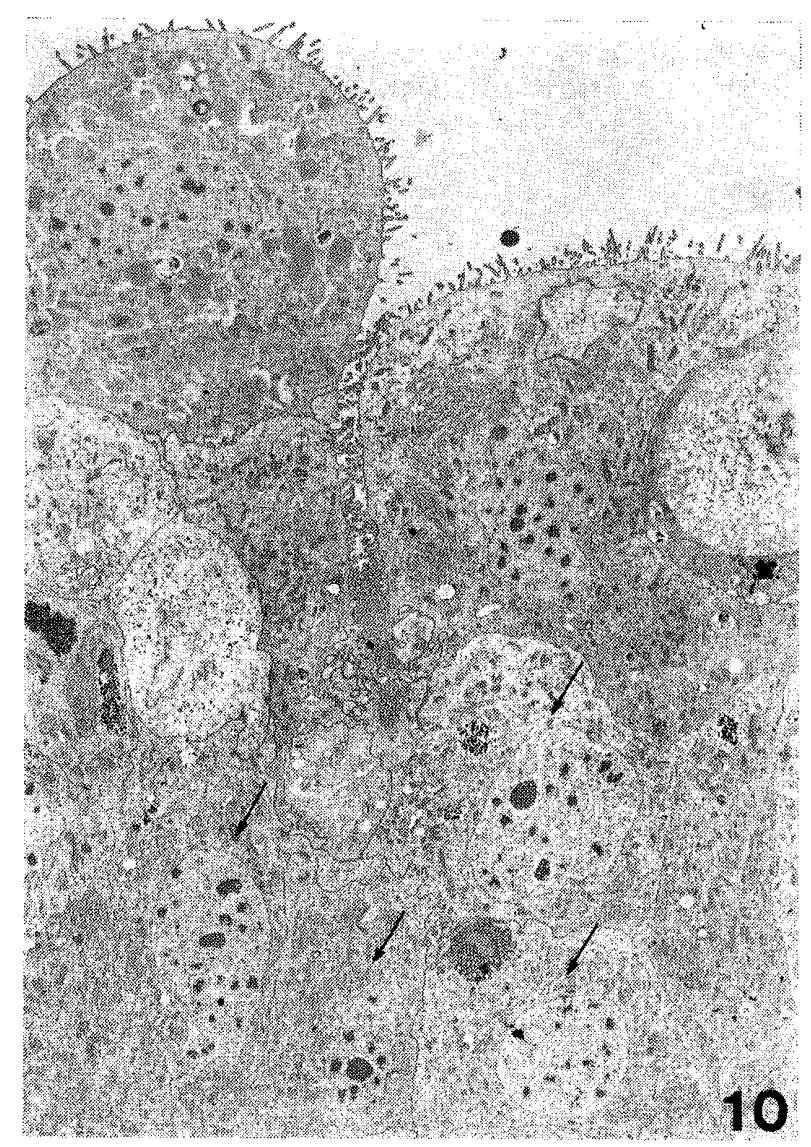

Fig. 10. Electron micrograph of upper portion of midgut epithelium at $24 \mathrm{hr}$ after the forced injection of crystals (200 $\mathrm{g}$ /larva). New epithelial cells (arrows) develop from the regenerative cells to replace the disintegrated cells. $\times 1,800$.

lumen, a number of crystals were found at the 4-hr stage. However, at this stage, a few crystals remained in the midgut and some were found in the hindgut, which would probably mean no gut paralysis occurred in Galleria larvae following the ingestion of the $\delta$-endotoxin.

Toxin-treated cells easily became detached from the basement lamina following the quick development of the regenerative cells. This was characteristic of Galleria. Since only a single administration of the toxin was done, the detached cells died and the new cells which replaced then were safe as the crystals had passed through the midgut lumen.

According to Hoopingarner and Materu (1964), under a light microscope Feulgen-positive nuclei were found only at the basal portion of the midgut epithelium in sixth-instar larvae of Galleria $4 \mathrm{hr}$ after the administration of the toxic diet containing $4 \%$ B.t. After $24 \mathrm{hr}$, destruction of the epithelial cells was extensively observed, and in the larvae surviving after 22 days, the epithelium was reduced to a thin cuboidal cell layer. Their observation seemed to correspond well with our observation. Their 24-hr stage would be our 4-hr stage. We found Galleria larvae is hardly killed by a single administration of the toxin even if the toxic dose is very high. When the toxic diet is constantly given, most of the larvae die in 3-4 days. These facts indicate that until the midgut epithelium becomes a thin cuboidal cell layer, cell regenera- 
Table 1. Changes in Hemolymph Following the Administration of $\delta$-Endotoxin Per Os into Sixth Instars of $G$. mellonella

\begin{tabular}{rcccccc}
\hline & \multirow{2}{*}{$\begin{array}{c}\text { *No. of } \\
\text { samples }\end{array}$} & $\mathrm{pH}$ & \multicolumn{4}{c}{$\mathrm{mEq} / 1$} \\
\cline { 4 - 7 } & & & $\mathrm{Na}^{+}$ & $\mathrm{K}+$ & $\mathrm{Mg}^{++}$ & $\mathrm{Ca}^{++}$ \\
\hline Control & 2 & $6.54 \pm 0.01$ & $15 \pm 1.0$ & $25 \pm 0.5$ & $37 \pm 2$ & $12 \pm 0$ \\
Toxin $4 \mathrm{hr}$ & 2 & $6.56 \pm 0.03$ & $14 \pm 0.2$ & $33 \pm 0.5$ & $30 \pm 3$ & $10 \pm 0.2$ \\
$24 \mathrm{hr}$ & 6 & $6.68 \pm 0.05$ & $13 \pm 0.8$ & $30 \pm 2.0$ & $23 \pm 1$ & $11 \pm 0.3$ \\
\hline Gut juice & pooled & $\mathbf{8 . 2 5}$ & 6 & 160 & 4.5 & 4.5 \\
\hline
\end{tabular}

* Either 200, 100 or $50 \mu \mathrm{g}$ of crystals per larva was injected per os. Two blood samples were collected and pooled from 7-8 larvae each. Since no difference in results was found, the value represents the mean of 6 samples at $4 \mathrm{hr}$ and $24 \mathrm{hr} \pm$ standard error of mean. Gut juice was collected and pooled from 100 normal larvae.

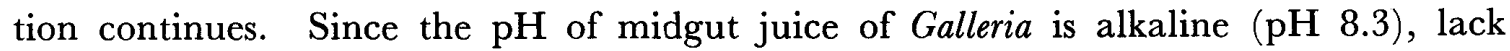
of some proteolytic enzyme(s) to dissolve the crystals might be also related to the low susceptibility to the toxin.

\section{Hemolymph chemistry}

Although two samples each (total 15 larvae) of 200, 100 and $50 \mu \mathrm{g}$ crystals injection were prepared at $4 \mathrm{hr}$ and $24 \mathrm{hr}$, we found hardly any difference in the results due to the injection dose. Table 1 summarized the results.

At $4 \mathrm{hr}$ after the forced injection of the pure crystals alone per os, the level of $\mathrm{K}^{+}$ in the hemolymph rose to 1.3 times that of normal blood (without further increase), while the blood $\mathrm{pH}$ scarcely changed. The $\mathrm{Mg}^{++}$concentration seemed to decrease gradually and slight fluctuations in the level of $\mathrm{Na}^{+}$and $\mathrm{Ca}^{++}$were observed those seen in Lymantria dispar (unpublished data). As mentioned above, at the histopathological point of view of the columnar cells of the midgut epithelium, the 4-hr stage of $G$. mellonella corresponded to stage 3 of completely paralyzed Bombyx. Changes appearing in Galleria hemolymph were slightly weaker than those in Lymantria, yet they were stronger than those in Ephestia, in which no change was found at the corresponding histopathological stages (unpublished data).

Thus, we again emphasize that there are sequential differences from species to species on any account of pathological changes induced by $\delta$-endotoxin.

\section{REFERENGES}

Burges, H. D., E. M. Thomson and R. A. LAtchford (1976) Importance of spores and $\delta$-endotoxin protein crystals of Bacillus thuringiensis in Galleria mellonella. J. Invertebr. Pathol. 27 : 87-94.

Delafield, F. P., H. J. Somerville and S. C. Rittenberg (1968) Immunological homology between crystal and spore protein of Bacillus thuringiensis. J. Bacteriol. 96 : 713-720.

Endo, Y. and J. Nishirsutsuji-Uwo (1980) Mode of action of Bacillus thuringiensis $\delta$-endotoxin: Histopathological changes in the silkworm midgut. J. Invertebr. Pathol. $36: 90-103$.

FAst, P. G. (1977) Bacillus thuringiensis $\delta$-endotoxin : On the relative roles of spores and crystals in toxicity to spruce budworm (Lepidoptera : Tortricidae). Canad. Entomol. 109 : 1515-1518.

Goodman, N. S., R. J. Gottrried and M. H. Rogoff (1967) Biphasic system for separation of spores and crystals of Bacillus thuringiensis. J. Bacteriol. $94: 485$.

Heimpel, A. M. and T. A. Angus (1959) The site of action of crystalliferous bacteria in Lepidoptera 
larvae. J. Insect Pathol. 1 : 152-170.

Hoopingarner, R. and M. E. A. Materu (1964) The toxicology and histopathology of Bacillus thuringiensis Berliner in Galleria mellonella (Linnaeus). J. Insect pathol. 6 : 26-30.

MaGaughey, Wm. H. (1978) Response of Plodia interpunctella and Ephestia cautella larvae to spores and parasporal crystals of Bacillus thuringiensis. J. Econ. Entomol. 71 :687-688.

Nishitssutsuji-Uwo, J. and Y. Endo (1980a) Mode of action of Bacillus thuringiensis $\delta$-endotoxin: General characteristics of intoxicated Bombyx larvae. J. Invertebr. Pathol. 35 : 219-228.

Nishirtsutsuji-Uwo, J. and Y. Endo (1980b) Mode of action of Bacillus thuringiensis $\delta$-endotoxin: Relative roles of spores and crystals in toxicity to Pieris, Lymantria and Ephestia larvae. Appl. Ent. Zool. 15 : 416-424.

Nishitsutsuji-Uwo, J., Y. Endo and M. Himeno (1979) Mode of action of Bacillus thuringiensis $\delta$ endotoxin: Effect on TN-368 cells. J. Invertebr. Pathol $34: 267-275$

Nishirtsutsuji-Uwo, J., Y. Wakisaka and M. EdA (1975) Sporeless mutants of Bacillus thuringiensis. J. Invertebr. Pathol. 25 : 355-361.

Nishimura, M. S. and J. Nishirtsutsuji-Uwo (1980) Sporeless mutants of Bacillus thuringiensis. III. The process of crystal formation. Tissue \& Cell $12: 233-241$.

SAcks, L. E. and G. Alderton (1961) Behavior of bacterial spores in aqueous polymer two-phase systems. J. Bacteriol. $82: 331-341$. 